\title{
The Evolutionary Trend in Computing and the Challenges This Presents to the Academic Environment
}

\author{
Marcel Ohanga Odhiambo \\ Department of Process Control and Computer Systems \\ Vaal University of Technology \\ Pretoria, South Africa
}

\author{
Weston Mwashita \\ Department of Process Control and Computer Systems \\ Vaal University of Technology \\ Pretoria, South Africa
}

\begin{abstract}
Cloud Computing and the use of the NETCOMPUTER have unlocked the door of high-tech education. The anytime-anywhere accessibility of information stored in distant cloud servers, has made it possible to seamlessly access courseware and educational materials from any device and from anywhere-anytime. Students can read and collaboratively work on some teacher/lecturer given assignment by use of any device and at any time. Besides the ease of access and collaboration, cloud-based education apps have solved the problem of storing data as well.
\end{abstract}

Keywords-Cloud Computing; NET-COMPUTER; Intelligent Agents; cloud-based interconnection; Academic Environment

\section{INTRODUCTION}

The computing trend has evolved over time, starting with mainframes, desktops, laptops, palmtops, iPads, notebooks, etc. All these trends, however, make use of local computing. Nevertheless, there is a shift or a move whereby, computing is not only remote but, computing resources are remotely hosted.

This has been brought about by the Information Communication Technology (ICT), which is the merging of computing activities such as document processing with communication, enabling one to prepare a document and communicate or post the document in one session. In addition, the ownership of computing resources and the use of the same are becoming very much separate. Hence, the supplier of computing resources is not necessarily the user at the same time. This has resulted in various entities offering computing resources which can be accessed over the Internet by any user from any place and anytime.

The concept to shift the computing resources to the Internet was proposed in [1] based on Intelligent Agents (software/computer programs) which freely roam the Internet (interconnected hosts) executing user tasks. The Intelligent Agents [2], [3] carry user privileges, rights and are the user's representatives on the Internet that execute tasks on behalf of the user. When the task terminates, the Intelligent Agents harvest the results and send these to the user. The combination of Intelligent Agents and the Internet gives rise to the concept of the NET-COMPUTER as outlined in [1]. Loosely explained, a NET-COMPUTER is a computer system in which the computing resources reside on the Internet and the user is provided with a simple interface to login to the Internet to access the computing resources.

Reliable computer usage statistics have shown that: (i) Nearly $90 \%$ of computer users, run document processing packages.

(ii) In an 8-hour working day, effective computer usage is about $40 \%$ in the office and $20 \%$ at home.

(iii) The ordinary computer user utilizes less than $10 \%$ of the computing resources e.g. processor functionalities.

(iv) The result is under usage of computing resources.

The proposition to address the stated computer usage is to:

(i) Concentrate computing resources on the Internet.

(ii) There is no need for powerful desktops, laptops, etc. since computing resources can safely reside on the Internet.

(iii) Provide the user with a simple, less complicated interface to access the Internet computing resources and run user tasks.

The NET-COMPUTER as such has not been physically implemented and tested but, the feasibility of Intelligent Agents executing in network computer was simulated in [4]. The proposed NET-COMPUTER based on intelligent agents and network hosts can be applied in Engineering and ECommerce, [5], [6], [7].

\section{CLOUD COMPUTING}

Cloud computing is a phrase used to describe a variety of computing concepts that involve many computers connected through a real-time communication network such as the Internet. Figure 1 [6] depicts the concept of cloud computing.

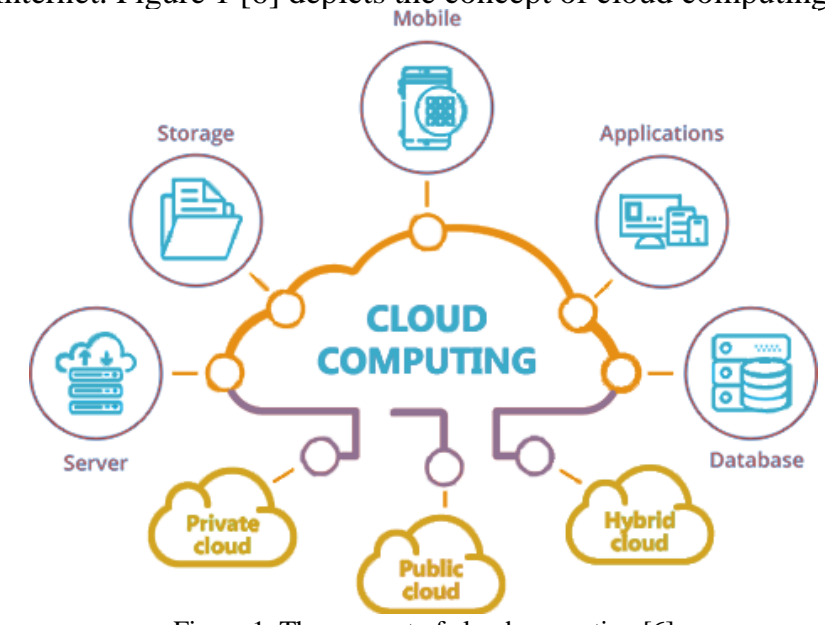

Figure 1: The concept of cloud computing [6] 
In science, cloud computing is synonym for distributed computing over a network and means the ability to run a program or application on many connected computers at the same time without knowing the physical location of the computers. Cloud computing is a computing term metaphor that evolved in the late 2000s, based on utility and consumption of computer resources. Cloud computing involves deploying groups of remote servers and software networks that allow centralized data storage and online access to computer services or resources. Clouds can be classified as public, private or hybrid [7], [8]. Cloud computing [9] relies on sharing of resources to achieve coherence and economies of scale, like a utility (like the electricity grid) over a network [8]. At the foundation of cloud computing is the broader concept of converged infrastructure and shared services.

Cloud computing, or in simpler terms, "the cloud", also focuses on maximizing the effectiveness of the shared resources. Cloud resources are usually not only shared by multiple users but are also dynamically reallocated per demand. This can work for allocating resources to users. For example, a cloud computer facility that serves European users during European business hours with a specific application (e.g., email) may reallocate the same resources to serve North American users during North America's business hours with a different application (e.g., a web server). This approach should maximize the use of computing power thus reducing environmental damage as well since less power, air conditioning, rack space, etc. are required for a variety of functions. With cloud computing, multiple users can access a single server to retrieve and update their data without purchasing licenses for different applications.

The term "moving to cloud" also refers to an organization moving away from a traditional CAPEX model (buy the dedicated hardware and depreciate it over a period of time) to the OPEX model (use a shared cloud infrastructure and pay as one uses it). Proponents of the technology claim that cloud computing allows companies to avoid upfront infrastructure costs and focus on projects that differentiate their businesses instead of on infrastructure [11]. Proponents also claim that cloud computing allows enterprises to get their applications up and running faster, with improved manageability and less maintenance and, enables IT departments to more rapidly adjust resources to meet fluctuating and unpredictable business demands [11], [12], [13]. Cloud providers typically use a "pay as you go" model. This can lead to unexpectedly high charges if administrators do not adapt to the cloud pricing model [13].

The present availability of high-capacity networks, lowcost computers and storage devices as well as the widespread adoption of hardware virtualization, service-oriented architecture, autonomic and utility computing, have led to a growth in cloud computing [14], 15], [16]. Companies can scale up as computing needs increase and then scale down again as demands decrease.
The phrase is often used in reference to network-based services, which appear to be provided by real server hardware and are in fact served by virtual hardware, simulated by software running on one or more real machines. Such virtual servers do not physically exist and can therefore be moved around and scaled up or down on the fly without affecting the end user, somewhat like a cloud becoming larger or smaller without being a physical object.

In common usage, the term "cloud computing" is essentially a metaphor for the Internet. Marketers have further popularized the phrase "in the cloud" to refer to software that is sold "as a service", i.e. remotely through the Internet. Typically, the seller has actual energy-consuming servers which host products and services from a remote location thus, end-users do not own; they simply log on to the network without installing anything. The major models are known as software as a service, platform as a service, and infrastructure as a service. These cloud services may be offered in a public, private, or hybrid network. Google, Amazon, Salesforce, and Microsoft Azure are some well-known cloud-vendors.

As a metaphor for the Internet, "the cloud" is a familiar cliché, but when combined with "computing", the meaning gets bigger and fuzzier. Some analysts and vendors define cloud computing narrowly as an updated version of utility computing: basically, virtual servers [16] available over the Internet. Others go very broad, arguing anything outside the firewall is "in the cloud", including conventional outsourcing.

Cloud computing comes into focus only when one considers what IT always needs: a way to increase capacity or add capabilities on the fly without investing in new infrastructure, training new personnel, or licensing new software. Cloud computing encompasses any subscription based or pay-per-use service that extends its existing capabilities.

Cloud computing is at an early stage, with a motley crew of providers, large and small, delivering a slew of cloud-based services, from full-blown applications to storage services and spam filtering. Yes, utility-style infrastructure providers are part of the mix, but so are SaaS (Software as a Service) providers. Today, for the most part, IT must plug into cloudbased services individually, but cloud computing aggregators and integrators are already emerging.

Today, with such cloud-based interconnection seldom in evidence, cloud computing might be more accurately described as "sky computing", with many isolated clouds of services which IT customers must plug into individually. On the other hand, as virtualization permeate the enterprise, the idea of loosely coupled services running on an agile, scalable infrastructure should eventually make every enterprise a node in the cloud. It is a long-running trend with a far-out horizon. But among big meta-trends, cloud computing is the hardest one to argue with. Cloud computing is thus an example of Internet computing though not based on Intelligent Agents. The inclusion of cloud computing is to provide an indication of the move towards Internet computing. 


\section{APPLICATIONS}

Cloud computing aims to provide computing resources in the cyber space or Internet in the form of virtual servers available over the Internet. Cloud computing relieves small and medium enterprises the headaches associated with the installation and maintenance of computing resources, cuts down on IT expenditure (personnel, infrastructure) and users only pay for the services/resources consumed.

Despite cloud computing social, legal, ownership, responsibility, availability, performance, compliance, cost, regulatory and many other issues, cloud computing has become a reality and promises to deliver computing services to various sectors of the business community. Nevertheless, security remains a major concern in cloud computing [5], [6], [7].

One commonly talked about cloud use case has been the shift to the inevitable online education - particularly the one rolled out by platforms like Microsoft Teams. Nada Ihab, Government Affairs Lead for Microsoft's Middle East \& Africa (MEA), outlined how by working closely with the United Arab Emirates (UAE)'s Telecommunications Regulatory Authority (TRA), Microsoft was recently able to connect slightly over a million students in the UAE to an entirely digital experience within 10 days.

\section{A. Cloud Computing in the Academic Environment}

Several organisations such as Amazon, Microsoft, Google, Pamoja, Vodafone Egypt, etc. offer cloud computing environment [8], [9], [10] and infrastructure and, academic institutions are already making use of these infrastructures. Cloud computing has extended to the academic space in which tuition content is offered online, enabling students to obtain qualifications online. Some of the educational content provided on the online platforms includes free content also referred to as Massive open online courses (MOOCS).

The online delivery of tuition is more beneficial for provision of open distance learning (ODL). The ODL pedagogy thrives on information technology, as it makes the interaction instant and the content accessible anytime, anywhere. This online delivery therefore requires extensive technology infrastructure to support the model and makes the content available. In this way, cloud computing is offering invaluable opportunities to learners to access educational materials online. Several establishments like University of South Africa, (UNISA) and Vaal University of Technology (VUT), offer study materials online. The infrastructural cost of offering the same can be very daunting to these organizations depending on their financial capability. Cloud computing technology in which the computing resources are held by a different organization can be used by only paying for time and the resources used, offers a more affordable access to learning opportunities.

Cloud computing offers the benefits of lowering infrastructural costs by residing the educational resources in the cloud. The institution offering the qualification does not necessarily have to meet the infrastructural cost. This could be a major saving in terms of operational as capital expenditures.

To the learners, cloud computing makes it possible to not only have access to one source of learning materials but to several sources offered by different institutions in the same field. Thus, there is a variety of available learning resources that the learners are exposed to.

Management of student's records is made more efficient particularly for academic institutions with different buildings and campuses scattered all over the country, where students tend to work from different places on the same day, or even spend a semester abroad. For instance, a printed academic file can be stored in a central archive and, different departments of the educational institution can access an online copy from any place in campus at any given time.

\section{B. Challenges of Cloud Computing (academic staff and students) in an Academic Environment)}

Nevertheless, this diversity of learning resources and the styles may present a dilemma to the learners in that it becomes the learner's responsibility to sift through the materials to pick what is relevant to their circumstances. This can at times be a big challenge to the learners especially those who are not Internet savvy. The result could be an overwhelming information overload for the learners. In addition, the learning resources are offered by different institutions in different formats and styles. Coupled with this, is the proliferation of devices used to access the learning resources. The question for the learners thus, becomes: which format, style, device, etc. is most suitable for learning?

The same challenges experienced by the learners are also experienced by the educators. As for educators, it's made much worse by the fact that most of them are not trained to prepare learning materials for Internet delivery, leave alone the ever-changing devices that are available in the market that learners can use to have access to the learning materials. It thus, becomes a question: are educators well equipped/trained to not only develop learning materials for Internet delivery but for the devices that are readily available to the learners?

Protocols have been developed by Cloud Computing suppliers to maintain confidentiality and safety of clients' data stored in their servers and, they provide additional warrantees and services to customers who need an additional safekeeping of information. This is however, the main concern that institutions have for not entering the cloud. The protocols offered by the Cloud Computing suppliers might provide sound internal security, a computer terminal might not have the same safety protocols. Therefore, a person might be reluctant to enter confidential information in a computer that can be used by anybody else.

There are fears about the safekeeping of confidential information in servers located in distant areas, specifically those countries where data protection regulation is not 
available. UNESCO has been made aware of these concerns, and there are arrangements to ensure personal data is only stored in determined countries with safe legal systems.

Having educational institution centralizing the management of its information in a specific cloud software makes it become overly dependent on that cloud software and any failure with a single supplier with a centralized system can be critical, thus UNESCO suggests that institutions diversify the number of software suppliers to mitigate risks.

Cloud Computing makes redundancy, where the same data is stored in more than one place become affordable to many institutions by storing information in a large pool of servers around the world. This helps guarantee a speedy access at any time and backs up data in case of any physical or digital problem with a server or if in any circumstance, the institution is threatened with the loss of critical information.

As depicted in Figure 2, students, trainees, teachers, lecturers and administrators can easily have access to vital information stored in servers scattered across the whole world from their computers in the comfort of their living/study rooms or offices without the installation of a specific programmes. This flexibility facilitates seamless interdepartmental collaboration. While one area supplies records to a common repository, another area can provide other records. At the same time forms, text files, presentations and spreadsheets can be edited simultaneously in real time by different people from any computer, helping in an efficient distribution of tasks and improving the quality of information by boosting peer feedback.

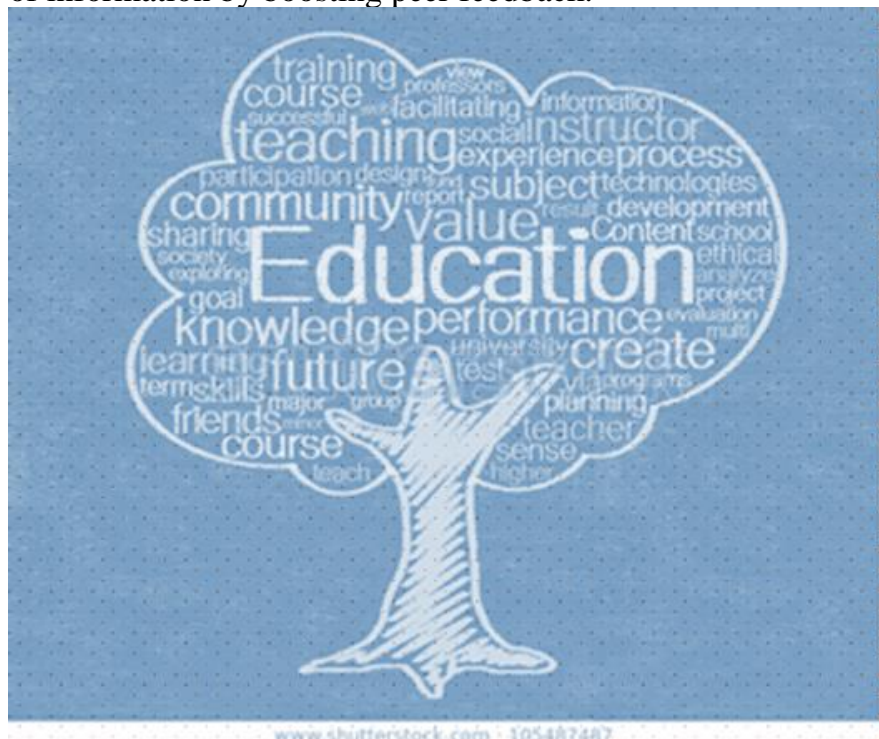

Figure 2: The "Cloud" [18]

\section{The way forward}

In cloud environment there is no one application which suits all requirements. It thus, becomes imperative that academic institutions need to make informed choices of what best units their operational needs for their clients (staff and students).

\section{CLOUD COMPUTING AND COVID-19}

The ravaging COVID-19 pandemic has gravitated humans into reinventing how they work, learn, interact, engage, and socialize. It has created an enabling environment for businesses and governments to quickly seek and thereafter apply solutions to respond to the crisis and maintain continuity. This has presented an unprecedented and pressing need to look for solutions to keep countries and their citizens running during COVID-19.

A similar pandemic cannot be completely ruled out soon so policymakers and regulators should focus on futureproofing; creating the right infrastructure to quickly deploy and adopt necessary cloud solutions. As Doreen Bogdan-Martin, Director, ITU BDT, puts it, "Cloud computing represents the catalyst and the enabler of the important technological shift that was already well underway before COVID-19" [17].

While educational institutions are required to provide more evidence and indicators of their academic quality to accreditation bodies nationally and internationally, Cloud Computing platforms facilitate easier registration and documentation. The educational institutional staff do not have to invest much time to gather, code and process large amounts of information but only to submit them into the system.

At the same time, this assists in keeping confidentiality in evaluations such as academic performance surveys and, in the gathering of external assessments, such as standardized English proficiency tests or higher education placement examinations. At the same time, the cloud's elasticity (which means that there is more capacity available whenever an institution needs it) can help in managing situations where the traffic of information increases dramatically - for instance, in periods of high demand for undergraduate or graduate applications.

\section{CONCLUSION}

Cloud computing is a reality that cannot be ignored. An academic institution ignoring cloud computing does so at their own peril. Despite the issues such as security, information integrity, legal, social, etc, associated with cloud computing, cloud computing offers a cost-effective means (without worrying about the infrastructure) for academic institutions not only to offer great learning opportunities for the learners but, also a means to diversify their qualification offering based on the fact that the learning materials are not only readily available in different formats, styles, etc, thus, providing the learners with realistic choices and a conducive learning environment in which to explore the knowledge boundaries.

\section{REFERENCES}

[1] M.O. Odhiambo, "Intelligent Agents: The Advent of the NetComputer". Kenya Engineer January/February 1999. A Journal of the Institution of Engineers of Kenya, 1999, pp. 32-35. 
[2] S. J. Russell and P. Norvig, "Artificial Intelligence: A Modern Approach", $4^{\text {th }}$ ed, Upper Saddle River, New Jersey, Prentice Hall, http://aima.cs.berkeley.edu/, Retrieved on 24 August.2020.

[3] Intelligent Agents. MIT Encyclopaedia http://www.aaai.org/aitopics/html/agebts.html, Retrieved on 30 August 2020.

[4] M.0. Odhiambo, "Net-Computer: Internet Computer Architecture based on Intelligent Agent", Worldcomp'11: The World Congress in Computer Science, Computer Engineering and Applied Computing (Internet Computing (ICOMP'11), ISBN 1-60132-186-4. July 18th 21st 2011. Las Vegas, USA. pp333-338. www.world-academy-ofscience.org

[5] M.O. Odhiambo, B. J, Van Wyk and M.A Van Wyk, "Application of HYDRA Computer to collect Status Information for Network Quality of Service. The 9th World Multi-Conference on Systemics, Cybernetics and Informatics. July 10th - 13th 2005, Orlando, Florida, USA. ISBN080-05-00-50-0M. Young, The Technical Writer's Handbook. Mill Valley, CA: University Science, 1989.

[6] Networks Ulimitted, Cloud Computing Trends for 2019, Colorado, 2019. https://www.networksunlimited.com/cloud-computing-trendsfor-2019/ Retrieved on 1 September 2020.

[7] [Public cloud, http://www.networkcomputing.com/public-cloud-techcenter, Retrieved on $1^{\text {st }}$ September 2020.

[8] Private cloud, http://http.networkcomputing.com/private-cloud-techcenter, Retrieved on $1^{\text {st }}$ September 2020.

[9] M. Ali, "Green Cloud on the Horizon," in Proceedings of the 1st International Conferenceon Cloud Computing (CloudCom), pp. 451459, December 2009.

[10] "What is Cloud Computing?" (http://aws.amazon.com/what-is-cloudcomputing/). Amazon Web Services. Retrieved on $2^{\text {nd }}$ September 2020.
[11] R. Baburajan, "The Rising Cloud Storage Market Opportunity Strengthens Vendors," infoTECH, August 24, 2011" (http://it.tmcnet.com/channels/cloud-storage/articles/211183-risingcloud-storage-market-opportunity-strengthens-vendors.html) It.tmcnet.com. 2011-08-24. Retrieved $2^{\text {nd }}$ September 2020.

[12] K. Oestreich, "Converged Infrastructure" (http://www.thectoforum.com/content/coverged-infrastructure-0). CTO Forum. Thectoforum.com. Retrieved on 28.08.2020..

[13] "Where's The Rub: Cloud Computing's Hidden Costs" (http://www.forbes.com/sites/centurylink/2014/02/27/wheres-the-rubcloud-computings-hidden-costs/) . 2014-02-27. Retrieved on $2^{\text {nd }}$ September 2020.

[14] "Cloud Computing: Clash of the clouds" (http://www.eceonomist.com/displaystory.cfm?story_id=14637206). The Economist. 2009-10-15. Retrieved on $2^{\text {nd }}$ September 2020.

[15] "Cloud Computing will be as influential as E-business" (http://gartner.com/it/page.jsp?id=707508). Gartner. Retrieved on $2^{\text {nd }}$ September 2020.

[16] Gruman, Galen. "What cloud computing really means" (http://www.infoworld.com/d/cloud-computing/what-cloud-computingreally-means-031). InfoWorld. Retrieved on $2^{\text {nd }}$ September 2020.

[17] How cloud computing has supported the COVID-19 response, ITU News, (https://news.itu.int/how-cloud-computing-has-supported-thecovid-19-response/) Retrieved on $13^{\text {th }}$ September 2020.

[18] https://www.u-planner.com/en/blog/beneficios-y-desafios-de-la-nubeen-gestion-de-la-educacion-superior/ Retrieved on $13^{\text {th }}$ September 2020 